\title{
Lack of association between methylenetetrahydrofolate reductase genetic polymorphisms and postmenopausal breast cancer risk
}

\author{
JASMINA ZIVA CERNE ${ }^{1}$, VIDA STEGEL ${ }^{2}$, KSENIJA GERSAK ${ }^{1}$ and SRDJAN NOVAKOVIC ${ }^{2}$ \\ ${ }^{1}$ Institute of Medical Genetics, Department of Obstetrics and Gynecology, University Medical Centre Ljubljana; \\ ${ }^{2}$ Department of Molecular Diagnostics, Institute of Oncology Ljubljana, 1000 Ljubljana, Slovenia
}

Received September 6, 2010; Accepted November 26, 2010

DOI: $10.3892 / \mathrm{mmr} .2010 .406$

\begin{abstract}
Published data on the association between methylenetetrahydrofolate reductase (MTHFR) gene polymorphisms and breast cancer risk are inconclusive. We investigated the independent and the combined effects of two commonly occurring polymorphisms, MTHFR $677 \mathrm{C}>\mathrm{T}$ (rs1801133) and MTHFR 1298A $>\mathrm{C}$ (rs1801131), as well as their interaction with the use of hormone replacement therapy (HRT), to determine their potential contribution to breast cancer risk. We studied 530 breast cancer cases and 270 controls of the same age and ethnicity participating in a case-control study of postmenopausal women. The duration of HRT use was ascertained through a postal questionnaire. Genotyping was conducted by TaqMan $^{\circledR}$ allelic discrimination. Adjusted odds ratios and 95\% confidence intervals were calculated using logistic regression. No significant association was observed between either the individual or the combined MTHFR genotypes and the risk of postmenopausal breast cancer. Additionally, no effects resulting from the interaction between MTHFR genotypes and HRT use were detected. Therefore, our data do not support the hypothesis that genetic variation in the MTHFR gene is implicated in the aetiology of postmenopausal breast cancer.
\end{abstract}

\section{Introduction}

Folates, a group of water-soluble B-vitamins, are important nutritional factors and play an integral role in maintaining DNA stability by regulating DNA biosynthesis, DNA repair and DNA methylation. In the form of 5,10-methylenetetrahydrofolate, folates are required for de novo synthesis of both purines and the pyrimidine nucleoside thymidine. In the form of 5-methyltetrahydrofolate, they are involved in the remethylation of homocysteine to methionine, the precursor

Correspondence to: Dr Srdjan Novakovic, Department of Molecular Diagnostics, Institute of Oncology Ljubljana, Zaloska 2, 1000 Ljubljana, Slovenia

E-mail: snovakovic@onko-i.si

Key words: breast cancer, folate, methylenetetrahydrofolate reductase of S-adenosylmethionine, which is the principal methyl donor in most cellular reactions (1).

Folate deficiency induces and accelerates carcinogenesis by perturbing each of these processes. Low dietary folate intake or impaired folate absorption or metabolism lead to purine and thymidine depletion and incorporation of uracil into DNA during DNA synthesis. The consequences are DNA strand breakage and chromosomal aberrations. Furthermore, cytosine methylation is altered, which may induce both gene-specific DNA hypermethylation and global DNA hypomethylation, potentially decreasing essential tumor-suppressor activation and increasing inappropriate proto-oncogene activation (2).

Polymorphisms in the genes involved in the transport of folate or its metabolism - thymidilate synthetase (TS), methylenetetrahydrofolate reductase (MTHFR), methionine synthase $(M T R)$ and methionine synthase reductase $(M T R R)$ - may result in allozymes with altered activity and are thus believed to cause interindividual differences in cancer risk susceptibility. It has been suggested that under conditions of limiting folate, one-carbon units are directed preferentially through the methionine cycle to facilitate methylation reactions at the expense of DNA synthesis and repair (3). MTHFR catalyzes the irreversible reduction of 5,10-methylenetetrahydrofolate to 5-methyltetrahydorfolate (Fig. 1) and is thus a critical enzyme redirecting the pool of folate from DNA synthesis and repair to DNA methylation (4).

Epidemiologic studies of breast cancer in association with MTHFR status have focused on two common gene variants. The most common variant of the MTHFR gene is $677 \mathrm{C}>\mathrm{T}$, which encodes a thermolabile variant of the enzyme with an alanine-to-valine substitution at position 222. The substitution results in a reduction of enzyme activity. Homozygotes (677TT) have approximately 30\% and heterozygotes (677CT) have approximately $65 \%$ of the activity of homozygous wildtypes (677CC), respectively (5). Another polymorphism of the MTHFR gene is $1298 \mathrm{~A}>\mathrm{C}$, which encodes a variant of the enzyme with a glutamate-to-alanine substitution at position 429 and has also been related to decreased enzyme activity (6). Homozygotes (1298CC) had approximately $60 \%$ of the activity of the homozygous wild-types (1298AA) (7).

The aim of the present study was to investigate the independent and the combined effects of two commonly occurring polymorphisms, MTHFR $677 \mathrm{C}>\mathrm{T}$ (rs1801133) and MTHFR 1298A >C (rs1801131), as well as their interaction 
with the use of hormone replacement therapy (HRT), to determine their potential contribution to breast cancer risk in postmenopausal women.

\section{Patients and methods}

Patients and controls. In this study, 530 postmenopausal women diagnosed with invasive primary breast cancer between January 1, 2006 and December 31, 2008 at the Institute of Oncology Ljubljana were enrolled. All were of Caucasian ethnic origin and aged between 50-69 years at the time of diagnosis. The control group consisted of 270 postmenopausal women randomly selected from the outpatient clinic records of the Department of Obstetrics and Gynaecology, University Medical Centre Ljubljana, that were of the same age and ethnicity, and had no history of breast cancer.

Women were invited to participate through a postal questionnaire. Overall response rates were $82.5 \%$ for cases and $73.2 \%$ for controls. Complete data for all variables considered in the multivariate model were available for $78.4 \%$ of cases and $70.9 \%$ of controls. Of these controls, $38.1 \%$ donated a blood sample. The number of cases included in genotype analyses was therefore proportionally decreased by random selection to gain the 2:1 ratio appropriate for case-control comparisons. The final analysis thus included 800 postmenopausal women: 530 cases and 270 controls.

In addition to general information (socioeconomic status, weight and height), data on reproductive factors (age at menarche, number of pregnancies, age at first delivery, number of deliveries, breastfeeding and age at menopause), exogenous hormone use [oral contraceptives (OC) and HRT], family history of breast or ovarian cancer (first-degree relatives), smoking and alcohol consumption were collected. OC and HRT use for $<1$ year was considered no use. Women were assumed to be postmenopausal if they had no periods for at least 12 months before the reference date or had undergone a bilateral oophorectomy.

Informed written consent was obtained from all women enrolled in the study. The study protocol was approved by the National Medical Ethics Committee of the Republic of Slovenia (No. 61/06/07).

Genotyping. In case patients, DNA was extracted from formalin-fixed paraffin-embedded normal breast tissues using the HP PCR Template Preparation kit (Roche Diagnostics $\mathrm{GmbH}$, Mannheim, Germany). In controls, genomic DNA was extracted from whole blood using the FlexiGene DNA kit 250 (Qiagen $\mathrm{GmbH}$, Hilden, Germany). Genotyping for the polymorphisms MTHFR $677 \mathrm{C}>\mathrm{T}$ and MTHFR $1298 \mathrm{~A}>\mathrm{C}$ was conducted by the TaqMan ${ }^{\circledR}$ allelic discrimination method. For MTHFR $677 \mathrm{C}>\mathrm{T}, 1.1 \%$ of the samples failed. For MTHFR $1298 \mathrm{~A}>\mathrm{C}, 0.9 \%$ of the samples failed. Samples that failed to be genotyped were scored as missing. Reliability was assessed by random selection of 5\% of samples in which all genotypes were confirmed by sequencing using the ABI PRISM 7000 sequence detection system (Applied Biosystems, Werterstadt, Germany). Concordance was $100 \%$ for all genotypes.

Statistical analyses. T-tests (for means) and Chi-square tests (for frequencies) were carried out to detect differences in

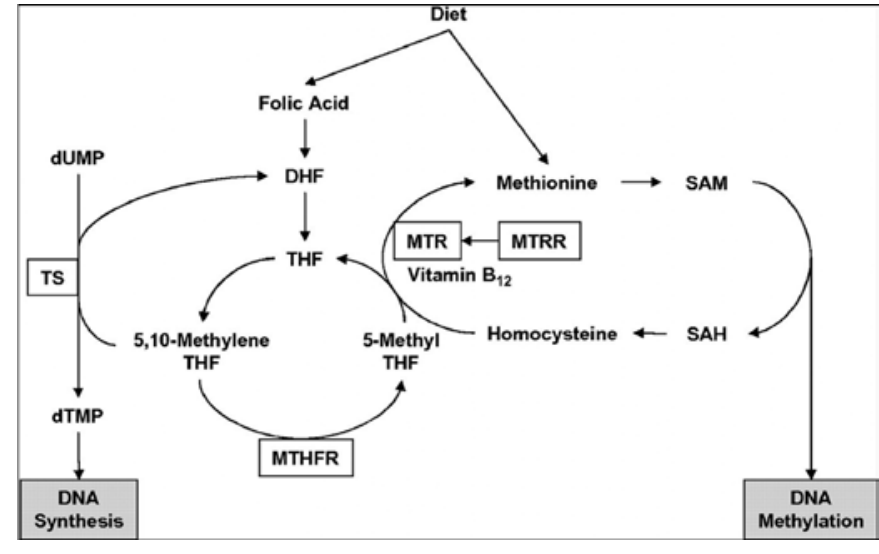

Figure 1. Folate metabolism [modified by Suzuki et al (4)]. THF, tetrahydrofolate; DHF, dihydrofolate; dUMP, deoxyuridine monophoshate; dTMP, deoxythymidine monophoshate; SAM, S-adenosylmethionine; SAH, S-adenosylhomocysteine; TS, thymidilate synthetase; MTHFR, methylenetetrahydrofolate reductase; MTR, methionine synthase; MTRR, methionine synthase reductase.

baseline characteristics between cases and controls. Observed genotype frequencies were tested for deviation from HardyWeinberg equilibrium with the Chi-square goodness-of-fit test. Odds ratios (ORs) for breast cancer risk and the corresponding 95\% confidence intervals (CI) were calculated using logistic regression analysis. The homozygous wild-type genotype, as determined by the more common of the homozygous genotypes, served as a reference category, with the heterozygous genotype and homozygous variant genotypes being collapsed into one category. Effect modification by the different genetic variants was investigated for the association between HRT use (never or $<1,1-5$ and $\geq 5$ years) and breast cancer risk. All analyses were adjusted by body mass index (BMI; $<25$, $25-30$ and $\geq 30 \mathrm{~kg} / \mathrm{m}^{2}$ ), years of OC use (never or $<1,1-5$, $5-10$ and $\geq 10$ years), years of HRT use (never or $<1,1-5$ and $\geq 5$ years) and smoking at the time of diagnosis (non-smokers, 1-10 cigarettes per day and $\geq 10$ cigarettes per day). Potential confounding effects of other known breast cancer risk factors were also examined, but none of those had a substantial effect on the ORs. A P-value of $<0.05$ was considered statistically significant. All statistical analyses were performed using SPSS 18.0 software package.

\section{Results}

Distribution of selected characteristics for cases and controls were consistent with most established risk factors and are presented in Table I. Rather unexpectedly, significantly more women in the control group were using HRT $(65.8 \%$ of controls vs. $29.6 \%$ of cases). Therefore, HRT use was highly significantly associated with a decrease in breast cancer risk ( $1-5$ years of HRT use: OR=0.22, 95\% CI $0.14-0.32 ; \geq 5$ years of HRT use: OR $=0.23,95 \% \mathrm{CI} 0.16-0.34)$. When adjusted for confounding effects, none of the two genetic variants (MTHFR $677 \mathrm{C}>\mathrm{T}$ and MTHFR $1298 \mathrm{~A}>\mathrm{C}$ ) was associated with breast cancer risk (Table II).

Next, we examined the possible combined effects of the MTHFR $677 \mathrm{C}>\mathrm{T}$ and MTHFR $1298 \mathrm{~A}>\mathrm{C}$ genotypes on breast 
Table I. Characteristics of the study population.

\begin{tabular}{|c|c|c|c|}
\hline Variable & Cases $(n=530)$ & Controls $(n=270)$ & P-value \\
\hline Education/highest degree obtained (\%) & & & $<0.001$ \\
\hline Primary school & 30.7 & 6.3 & \\
\hline Secondary school & 59.2 & 70.3 & \\
\hline University, $\mathrm{PhD}$ & 10.1 & 23.4 & \\
\hline $\operatorname{BMI}(\%)^{\mathrm{a}}$ & & & $<0.001$ \\
\hline$<25 \mathrm{~kg} / \mathrm{m}^{2}$ & 33.6 & 52.2 & \\
\hline $25-30 \mathrm{~kg} / \mathrm{m}^{2}$ & 40.7 & 35.1 & \\
\hline$\geq 30 \mathrm{~kg} / \mathrm{m}^{2}$ & 25.7 & 12.7 & \\
\hline Mean \pm SD age at menarche (years) & $13.7 \pm 1.8$ & $13.5 \pm 2.1$ & 0.021 \\
\hline Mean \pm SD age at spontaneous menopause (years) & $50.5 \pm 3.7$ & $50.4 \pm 3.9$ & 0.711 \\
\hline Nulliparity (\%) & 5.3 & 3.4 & 0.089 \\
\hline Mean \pm SD number of full-term pregnancies ${ }^{b}$ & $1.8 \pm 0.9$ & $1.7 \pm 0.9$ & 0.127 \\
\hline Mean \pm SD age at first delivery (years) ${ }^{b}$ & $24.0 \pm 4.6$ & $24.9 \pm 4.8$ & 0.012 \\
\hline Women that breastfed $(\%)$ & 86.4 & 90.3 & 0.344 \\
\hline Mean \pm SD duration of breastfeeding (months) ${ }^{c}$ & $8.1 \pm 8.7$ & $7.7 \pm 7.4$ & 0.269 \\
\hline OC use $(\%)$ & 42.1 & 54.7 & 0.001 \\
\hline Duration of OC use (\%) & & & 0.002 \\
\hline $0<1$ year & 57.9 & 45.3 & \\
\hline $1-5$ years & 14.4 & 22.7 & \\
\hline $5-10$ years & 12.7 & 17.1 & \\
\hline$\geq 10$ years & 15.0 & 14.9 & \\
\hline HRT use $(\%)$ & 29.6 & 65.8 & $<0.001$ \\
\hline Duration of HRT use (\%) & & & $<0.001$ \\
\hline $0<1$ year & 70.4 & 34.2 & \\
\hline $1-5$ years & 14.3 & 33.1 & \\
\hline$\geq 5$ years & 15.3 & 32.7 & \\
\hline Regimen of HRT $(\%)^{\mathrm{d}}$ & & & 0.487 \\
\hline Combined, estrogen plus progestin & 71.2 & 67.8 & \\
\hline Estrogen only & 28.8 & 32.2 & \\
\hline First degree family history of breast or ovarian cancer $(\%)$ & 18.1 & 15.5 & 0.138 \\
\hline Smoking (\%) & 20.2 & 15.9 & 0.041 \\
\hline
\end{tabular}

aCalculated as weight in kilograms divided by height in meters squared at the age of the diagnosis. ${ }^{\mathrm{b} A m o n g}$ women who had a full term pregnancy. ${ }^{c}$ Among those who ever breastfed. dAmong those who ever used HRT.

cancer risk. The reference group consisted of individuals with the putatively most advantageous combinations of the genotypes: low-risk genotypes, i.e., the presence of the homozygous CC genotype for MTHFR 677C>T and the AA genotype for MTHFR 1298A>C. An association between the combined MTHFR genotypes and breast cancer risk was also not detected (Table III).

Additionally, we investigated whether the MTHFR $677 \mathrm{C}>\mathrm{T}$ and MTHFR $1298 \mathrm{~A}>\mathrm{C}$ genotypes in interaction with HRT use (never or $<1,1-5$ and $\geq 5$ years) affect the risk of postmenopausal breast cancer. No interaction effects between MTHFR genotypes and HRT use were found (Table IV). Additionally, no interaction was observed between the genetic variants and breast cancer risk with respect to HRT regimen (estrogen monotherapy vs. combined, estrogen plus progestin therapy) (data not shown).

\section{Discussion}

In this case-control study of postmenopausal women we found no association between either the independent or the combined effects of two commonly occurring polymorphisms, MTHFR 677C $>$ T and MTHFR 1298A >C, and breast cancer.

Previous attempts to explore the relationship between these two polymorphisms and breast cancer have yielded conflicting results $(4,8,9,10)$, although two recent meta-analyses, involving 15.260 cases and 20.411 controls, and 16.480 cases and 22.388 controls, concluded that the functional MTHFR $677 \mathrm{C}>\mathrm{T}$ polymorphism may play a low penetrance role in the development of breast cancer $(11,12)$. By contrast, a clear inverse association has been consistently observed between the MTHFR 677TT genotype and colorectal cancer, especially with high levels of folate intake and low levels of alcohol intake (13). It has 
Table II. Genetic variation in MTHFR gene and risk of postmenopausal breast cancer.

\begin{tabular}{lccc}
\hline Genotype & Cases, $\mathrm{n}(\%)$ & Controls, $\mathrm{n}(\%)$ & OR $(95 \% \mathrm{CI})^{\mathrm{a}}$ \\
\hline MTHFR $677 \mathrm{C}>\mathrm{T}$ & & & 1.0 \\
CC & $222(42.5)$ & $108(40.1)$ & $0.95(0.67-1.35)$ \\
CT & $238(45.6)$ & $124(46.1)$ & $0.88(0.52-1.50)$ \\
TT & $62(11.9)$ & $37(13.8)$ & $0.94(0.68-1.31)$ \\
CT/TT & $300(57.5)$ & $161(59.9)$ & 0.792 \\
MTHFR 1298Alue & & 0.642 \\
AA $>C$ & $258(49.2)$ & $131(48.7)$ & $0.89(0.63-1.26)$ \\
AC & $219(41.8)$ & $117(43.5)$ & $1.04(0.57-1.92)$ \\
CC & $47(9.0)$ & $21(7.8)$ & $0.91(0.66-1.27)$ \\
AC/CC & $266(50.8)$ & $138(51.3)$ & 0.510 \\
\hline
\end{tabular}

${ }^{a}$ Adjusted for BMI $\left(<25,25-30\right.$ and $\left.\geq 30 \mathrm{~kg} / \mathrm{m}^{2}\right)$; years of OC use (never or $<1,1-5,5-10$ and $\geq 10$ ); years of HRT use (never or $<1,1-5$ and $\geq 5$ ); smoking (non-smokers, $1-10$ cigarettes per day and $\geq 10$ cigarettes per day).

Table III. Combined effects of MTHFR 677C $>$ T and MTHFR $1298 \mathrm{~A}>\mathrm{C}$ genotypes and risk of postmenopausal breast cancer.

\begin{tabular}{lcccc}
\hline \multicolumn{2}{c}{ Genotype } & Cases/Controls & OR $(95 \% \text { CI })^{\mathrm{a}}$ & \\
\hline MTHFR 677C $>\mathrm{T}$ & MTHFR $1298 \mathrm{~A}>\mathrm{C}$ & & \\
\hline CC & $\mathrm{AA}$ & $66 / 26$ & 1.0 & 0.164 \\
CC & $\mathrm{AC} / \mathrm{CC}$ & $156 / 82$ & $0.66(0.37-1.18)$ & 0.186 \\
CT/TT & $\mathrm{AA}$ & $191 / 105$ & $0.68(0.39-1.20)$ & 0.290 \\
CT/TT & $\mathrm{AC} / \mathrm{CC}$ & $109 / 56$ & $0.72(0.39-1.32)$ & \\
\hline
\end{tabular}

aAdjusted for BMI $\left(<25,25-30\right.$ and $\left.\geq 30 \mathrm{~kg} / \mathrm{m}^{2}\right)$; years of OC use (never or $<1,1-5,5-10$ and $\geq 10$ ); years of HRT use (never or $<1,1-5$ and $\geq 5$ ); smoking (non-smokers, $1-10$ cigarettes per day and $\geq 10$ cigarettes per day).

Table IV. HRT use, genetic variation in MTHFR gene and risk of postmenopausal breast cancer.

\begin{tabular}{|c|c|c|c|c|c|c|}
\hline \multirow[t]{3}{*}{ Genotype } & \multicolumn{6}{|c|}{ HRT use (years) } \\
\hline & \multicolumn{2}{|c|}{0 to $<1$} & \multicolumn{2}{|c|}{$1-5$} & \multicolumn{2}{|c|}{$\geq 5$} \\
\hline & Cases/Controls & $\mathrm{OR}^{\mathrm{a}}(95 \% \mathrm{CI})$ & Cases/Controls & $\mathrm{OR}^{\mathrm{a}}(95 \% \mathrm{CI})$ & Cases/Controls & $\mathrm{OR}^{\mathrm{a}}(95 \% \mathrm{CI})$ \\
\hline \multicolumn{7}{|c|}{ MTHFR $677 \mathrm{C}>\mathrm{T}$} \\
\hline $\mathrm{CC}$ & $156 / 38$ & 1.0 & $38 / 34$ & $0.29(0.16-0.53)$ & $28 / 36$ & $0.18(0.09-0.33)$ \\
\hline $\mathrm{CT} / \mathrm{TT}$ & $210 / 54$ & $0.95(0.59-1.53)$ & $38 / 55$ & $0.16(0.09-0.29)$ & $52 / 52$ & $0.28(0.16-0.48)$ \\
\hline $\mathrm{P}_{\text {interaction }}$ & 0.084 & & & & & \\
\hline \multicolumn{7}{|c|}{ MTHFR $1298 \mathrm{~A}>\mathrm{C}$} \\
\hline AA & $177 / 44$ & 1.0 & $35 / 45$ & $0.19(0.11-0.35)$ & $46 / 42$ & $0.29(0.16-0.50)$ \\
\hline $\mathrm{AC} / \mathrm{CC}$ & $190 / 48$ & $0.95(0.59-1.52)$ & $41 / 44$ & $0.23(0.13-0.40)$ & $35 / 46$ & $0.19(0.11-0.34)$ \\
\hline $\mathrm{P}_{\text {interaction }}$ & 0.447 & & & & & \\
\hline
\end{tabular}

${ }^{a}$ Adjusted for BMI ( $<25,25-30$ and $\geq 30 \mathrm{~kg} / \mathrm{m}^{2}$ ); years of OC use (never or $<1,1-5,5-10$ and $\geq 10$ ); years of HRT use (never or $<1,1-5$ and $\geq 5$ ); smoking (non-smokers, $1-10$ cigarettes per day and $\geq 10$ cigarettes per day).

been suggested that this is in agreement with the markedly lower rates of cell division and thus lower need for nucleic acid synthesis in the postmenopausal breast compared to large bowel (14). 
A study by Marchand et al found that the MTHFR 677TT genotype may confer a $40 \%$ decreased breast cancer risk in postmenopausal women using HRT (14). This is consistent with the need for increased nucleic acid resulting from the hyperproliferative effect of HRT on mammary epithelial cells and reduced MTHFR activity, which increases the flow towards DNA synthesis. Similarly, the weak inverse association between the MTHFR 677CT/TT and MTHFR 1298AC/ $\mathrm{CC}$ genotypes and breast cancer in our study was more pronounced among women on HRT. However, the test for interaction of the MTHFR $677 \mathrm{C}>\mathrm{T}$ and MTHFR $1298 \mathrm{~A}>\mathrm{C}$ polymorphisms and HRT use (never or $<1,1-5$ and $\geq 5$ years) on the risk of breast cancer did not reach statistical significance $\left(\mathrm{P}_{\text {interaction }} 0.084\right.$ and 0.447 , respectively). Whether there is any relationship between estrogens and folates in breast cancer remains to be determined. HRT is known to reduce plasma homocysteine concentrations, but independently of the MTHFR 677C $>$ T polymorphism (15).

Several limitations need to be considered when interpreting the results. The analysis revealed no indication of an increased risk of breast cancer with HRT use, which is inconsistent with previous studies suggesting that HRT use is associated with a small, but significant increase in the risk of breast cancer (16). Since a low percentage of control group women agreed to provide a blood sample, the extremely opposite trend in the present study might be due to preferential participation in the study by controls with this breast cancer risk factor (HRT use) present over those without this factor. Another explanation might involve the use of retrospectively collected exposure data since, as in most observational studies, we relied on selfreports of HRT use. For this reason, a colour chart displaying all preparations ever marketed in Slovenia was included in the questionnaire to aid recall. Furthermore, women of higher socioeconomic status are more than three times more likely to undergo HRT (17). In our study population, $10.1 \%$ of cases and $23.4 \%$ of controls reported having a university degree or $\mathrm{PhD}$, whereas $30.7 \%$ of cases and $6.3 \%$ of controls gave their highest level of education obtained as being primary school. Thus, given the higher education level and higher prevalence of HRT use among controls, we believe that the comprehensive medical care received by HRT users in whom no pre-existing breast abnormalities have been found by mammography examinations may explain the observed decrease in breast cancer risk with HRT use of our study.

Our study population was of medium size, and it is possible that some interactions were also not significant due to insufficient power. However, the study provides adequate power to detect clinically relevant interactions, with an $\mathrm{OR}=2.0$, assuming $\alpha=0.05$.

The strengths of the study include the availability of information on potential confounders and the investigation of functionally relevant genetic variants. Furthermore, for both polymorphisms assessed, the distribution of each genotype followed Hardy-Weinberg equilibrium, which indicates that no selection bias occurred among genotypes. Additionally, the study population was largely homogenous. Analyses were restricted to postmenopausal Slovenian Caucasian women; mean ages for cases and for controls did not differ significantly between the groups $(\mathrm{p}=0.432)$.
In conclusion, we report that breast cancer risk is unlikely to be influenced by functionally relevant variants in the MTHFR gene.

\section{Acknowledgements}

This study was supported by grant L3-0431 from the Slovenian Research Agency.

\section{References}

1. Kim YI: Folate and carcinogenesis: evidence, mechanisms, and implications. J Nutr Biochem 10: 66-88, 1999.

2. Duthie SJ: Folate and cancer: how DNA damage, repair and methylation impact on colon carcinogenesis. J Inherit Metab Dis: June, 2010 (E-pub ahead of print).

3. Kim YI: Folate and colorectal cancer: an evidence-based critical review. Mol Nutr Food Res 51: 267-292, 2007.

4. Suzuki T, Matsuo K, Hirose K, et al: One-carbon metabolismrelated gene polymorphisms and risk of breast cancer. Carcinogenesis 29: 356-362, 2008.

5. Frosst P, Blom HJ, Milos R, et al: A candidate genetic risk factor for vascular disease: a common mutation in methylenetetrahydrofolate reductase. Nature Genetics 10: 111-113, 1995.

6. Weisberg I, Tran P, Christensen B, Sibani R and Rozen R: A second polymorphism in methylenetetrahydrofolate reductase (MTHFR) associated with decreased enzyme activity. Mol Genet Metab 64: 169-172, 1998.

7. Van der Put NM, Gabreels F, Stevens EM, et al: A second common mutation in the methylenetetrahydrofolate reductase gene: an additional risk factor for neural-tube defects? Am J Med Genet 62: 1044-1051, 1998.

8. Maruti SS, Ulrich CM, Jupe ER and White E: MTHFR C677T and postmenopausal breast cancer risk by intakes of one-carbon metabolism nutrients: a nested case-control study. Breast Cancer Res 11: R91, 2009.

9. Kotsopoulos J, Zhang WW, Zhang S, et al: Polymorphisms in folate metabolizing enzymes and transport proteins and the risk of breast cancer. Breast Cancer Res Treat 112: 585-593, 2008.

10. Ericson U, Sonestedt E, Ivarsson MI, Gullberg B, Carlson J, Olsson $\mathrm{H}$ and Wirfält E: Folate intake, methylenetetrahydrofolate reductase polymorphisms, and breast cancer risk in women from the Malmö Diet and Cancer cohort. Cancer Epidemiol Biomarkers Prev 18: 1101-1110, 2009.

11. Zhang J, Qiu LX, Wang ZH, et al: MTHFR C677T polymorphism associated with breast cancer susceptibility: a meta-analysis involving 15,260 cases and 20,411 controls. Breast Cancer Res Treat 123: 549-555, 2010.

12. Qi X, Ma X, Yang X, et al: Methylenetetrahydrofolate reductase polymorphism and breast cancer risk: a meta-analysis from 41 studies with 16,480 cases and 22,388 controls. Breast Cancer Res Treat 123: 499-506, 2010.

13. Choi SW and Mason JB: Folate status: effects on pathways of colorectal carcinogenesis. J Nutr 132: 2413-2418, 2002.

14. Le Marchand L, Haiman CA, Wilkens LR, Kolonel LN and Henderson BE: MTHFR polymorphisms, diet, HRT and breast cancer risk: the Multiethnic Cohort Study. Cancer Epidemiol Biomarkers Prev 13: 2071-2077, 2004.

15. Somekawa Y, Kobayashi K, Tomura S, Aso T and Hamaguchi H: Effects of hormone replacement therapy and methylenetetrahydrofolate reductase polymorphism on plasma folate and homocysteine levels in postmenopausal Japanese women. Fertil Steril 77: 481-486, 2002.

16. Breast cancer and hormone replacement therapy: collaborative reanalysis of data from 51 epidemiological studies of 52,705 women with breast cancer and 108,411 women without breast cancer: Collaborative Group on Hormonal Factors in Breast Cancer. Lancet 350: 1047-1059, 1997.

17. Finley C, Gregg EV, Solomon LJ and Gay E: Disparities in hormone replacement therapy use by socioeconomic status in a primary care population. J Community Health 26: 39-50, 2001. 